\title{
Adomain Decomposition Method for Solving Non Linear Partial Differential Equations
}

\author{
Fadhil H. Easif \\ Department of Mathematics, Faculty of Science, University of Zakho,Duhok, Kurdistan Region, Iraq
}

\begin{abstract}
In this paper, an application of A domain Decomposition method (ADM) is applied for finding the approximate solution of nonlinear partial differential equation. The results reveal that the A domain Decomposition method is very effective, simple and very close to the exact solution.
\end{abstract}

Keywords: - A domain Decomposition method, nonlinear partial differential equation.

\section{Introduction}

It is well known that most of the phenomena that arise in mathematical physics and engineering fields can be described by partial differential equations (PDEs). In physics for example, the heat flow and the wave propagation phenomena are well described by partial differential equations [1,2]. In ecology, most population models are governed by partial differential equations $[5,6]$. The dispersion of a chemically reactive material is characterized by partial differential equations. In addition, most physical phenomena of fluid dynamics, quantum mechanics, electricity, plasma physics, propagation of shallow water waves, and many other models are controlled within its domain of validity by partial differential equations. Partial differential equations have become a useful tool for describing these natural phenomena of science and engineering models. Therefore, it becomes increasingly important to be familiar with all traditional and recently developed methods for solving partial differential equations, and the implementation of these methods.[3]

It is probably not an overstatement to say that almost all partial differential equations (PDEs) that arise in a practical setting are solved numerically on a computer.[4]

Numerical analysis is concerned with the development and investigation of constructive methods for numerical solution of mathematical problems. A numerical method is useful only if it is possible to decide accuracy of the approximate solution, i.e., if reliable estimates on the difference between the exact and approximate solution can be given. Therefore, besides the development and design of numerical schemes, a substantial part of numerical analysis is concerned with the investigation and estimation of the errors occurring in these schemes. Here one has to discriminate between the approximate errors, i.e., the errors that arise through replacing the original problem by an approximate problem, and the round off errors.[5]

Nonlinear partial differential equations are useful in describing the various phenomena in many disciplines. Apart of a limited number of these problems, most of them do not have a precise analytical solution, so these nonlinear equations should be solved using approximate methods.

This method starts by using the constant function as an approximation to a solution. We substitute this approximation into the right side of the given equation and use the result as a next approximation to the solution. Then we substitute this approximation into the right side of the given equation to obtain what we hope is a still better approximation and we continuing the process. Our goal is to find a function with the property that when it is substituted in the right side of the given equation the result is the same function. This procedure is known as successive approximation method Nowadays engineers and scientists in all fields of their research are using partial differential equations to describe their problems and thus such partial differential equations arise in the study of heat transfer, boundary-layer flow, fluid flow problems, vibrations elasticity, circular and rectangular wave guides, in applied mathematics and so on.[1]

Many physical, chemical and engineering problems mathematically can be modeled in the form of system of partial difference equations or system of ordinary difference equations. Finding the exact solution for the above problems which involve partial differential equations is difficult in some cases. Hence we have to find the numerical solution of these problems using computers which came into existence. [2]

Most problems and scientific phenomena, such as heat transfer, fluid mechanics, plasma physics, plasma waves, thermo-elasticity and chemical physics, occur nonlinearly. Except for a limited number of these problems, we encounter difficulties in finding their exact analytical solutions. Very recently, some promising approximate analytical solutions are proposed.[6]

Numerical methods were first put into use as an effective tool for solving partial differential equations (PDEs) by John von Neumann in the mid-1940s. [7]Numerical analysis is the branch of mathematics that is used to find approximations to difficult problems such as finding the roots of non-linear equations, integration involving complex expressions and solving differential equations for which analytical solutions do not exist. It 
is applied to a wide variety of disciplines such as business, all fields of engineering, computer science, education, geology, meteorology, and others. Years ago, high-speed computers did not exist, and if they did, the largest corporations could only afford them; consequently, the manual computation required lots of time and hard work. But now that computers have become indispensable for research work in science, engineering and other fields, numerical analysis has become a much easier and more pleasant task.[8]

The study of numerical methods for the solution of nonlinear partial differential equations has enjoyed an intense period of activity over the last 40 years from both theoretical and practical points of view. Improvements in numerical techniques, together with the rapid advances in computer technology, have meant that many of the partial differential equations arising from engineering and scientific applications.[9]

\section{II.1 Basic idea of Adomain Decomposition Method (ADM)}

\section{Indentations and Equations}

Consider the differential equation

$$
\begin{aligned}
& L u+R u+N u=g . \\
& u(x, 0)=f(x)
\end{aligned}
$$

Where $L$ is the operator of the highest-ordered derivatives and $R$ is the remainder of the linear operator. The nonlinear term is represented by $N(u)$.

Thus we get:

$$
\begin{array}{ll} 
& L u=g-R u-N u . \\
\text { Where } & L=\frac{\partial}{\partial t}, \text { define } L^{-1}=\int_{0}^{t}(.) d t .
\end{array}
$$

Operating with the operator $L^{-1}$ on both sides of Eq. (1) we have:

$$
\begin{aligned}
u=u_{0}-L^{-1}(R u)- & L^{-1}(N u) \\
& =f(x)-L^{-1}(R u)-L^{-1}(N u) .
\end{aligned}
$$

Where $L^{-1}(g)=u_{0}=u(x, 0)=f(x)$.

The standard Adomain decomposition method is define the solution $u(x, t)$ as an infinite series of the form:

$$
u(x, t)=\sum_{k=0}^{\infty} u_{k}(x, t)
$$

Where

$$
u_{0}(x, t)=u(x, 0)=f(x)
$$

$$
\begin{aligned}
& u_{1}(x, t), u_{2}(x, t), \ldots \text { are determin } \\
& u_{k+1}=-L^{-1}\left(R u_{k}\right)-L^{-1}\left(N u_{k}\right) \quad, k=1,2, \ldots
\end{aligned}
$$

And the nonlinear operator $N(u)$ can be decomposed by an infinite series of polynomials given by:

Where

$$
N(u)=\sum_{k=0}^{\infty} A_{k}
$$

$$
A_{k}=\frac{1}{k !} \frac{d^{k}}{d \lambda^{i}}\left[F\left(\sum_{i=0}^{k} \lambda^{i} u_{i}\right)\right]_{\lambda=0}, k=0,1, \ldots
$$

It is now well known in the literature that these polynomials can be constructed for all classes of nonlinearity according to algorithms set by Adomian [1,2] and recently developed by an alternative approach in [1-3].

\section{Figures And Tables}

We will apply Adomain decomposition method (ADM) to solve the nonlinear partial differential equations, and present numerical results to verify the effectiveness of this method, we take the following examples:

\section{Example 1: [6]}

$\frac{\partial^{2} u}{\partial t^{2}}=-\alpha \frac{\partial^{2} u}{\partial x^{2}}-\beta u-\gamma u^{3} \quad, t>0$

With the initial conditions

$u(x, 0)=B \tan (K x), u_{t}=B c K \sec ^{2}(K x) \quad,-1 \leq x \leq 1$

We take

$$
\alpha=-2.5, \beta=1, \gamma=1.5 \text {. Where }
$$

$$
B=\sqrt{\frac{\beta}{\gamma}} \text { and } K=\sqrt{\frac{-\beta}{2\left(\alpha+c^{2}\right)}}
$$




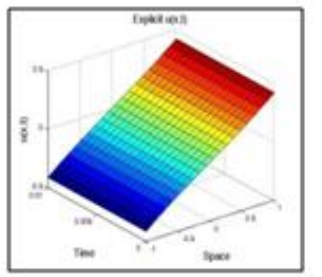

Fig. (1) FDM solution te 0 ete 0.01 And $-1<x<1$

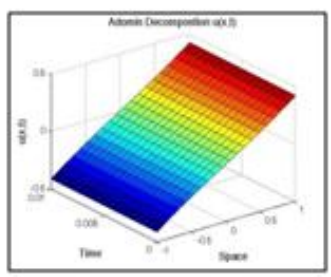

Fig. (2) ADM solution to 0 ete 0.01 and $-1<x<1$

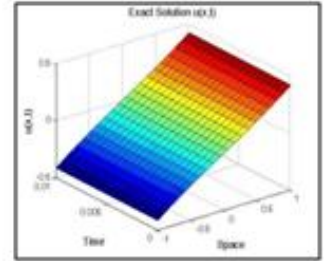

Fig. (3) Exact solution to 0 cte 0.01 and $-1<<<1$

Table (1) comparison between ADM, EFDM and Exact solutions at $\mathrm{t}=2$

\begin{tabular}{|c|c|c|}
\hline EXACT & $\mathrm{ADM}$ & EFDM \\
\hline-0.414411214133078 & -0.414411214101644 & -0.414411214133078 \\
\hline-0.364670241057009 & -0.364670241031911 & -0.364669012962060 \\
\hline-0.317087953610942 & -0.317087953590899 & -0.317086928637545 \\
\hline-0.271306324156148 & -0.271306324140212 & -0.271305477633181 \\
\hline-0.227011127968345 & -0.227011127955811 & -0.227010440389676 \\
\hline-0.183922496540239 & -0.183922496530586 & -0.183921952741587 \\
\hline-0.141787250668732 & -0.141787250661578 & -0.141786839088029 \\
\hline-0.100372541039790 & -0.100372541034864 & -0.100372253179315 \\
\hline-0.059460438539449 & -0.059460438536567 & -0.059460268585494 \\
\hline-0.018843195109709 & -0.018843195108769 & -0.018843139679920 \\
\hline 0.021681051104011 & 0.021681051103044 & 0.021680993104744 \\
\hline 0.062312318757989 & 0.062312318755080 & 0.062312146183729 \\
\hline 0.103252748218675 & 0.103252748213720 & 0.103252457634093 \\
\hline 0.144710656400331 & 0.144710656393147 & 0.144710241934291 \\
\hline 0.186904856153030 & 0.186904856143345 & 0.186904309243295 \\
\hline 0.230069434484844 & 0.230069434472276 & 0.230068743494559 \\
\hline 0.274459218894519 & 0.274459218878543 & 0.274458368570347 \\
\hline 0.320356215567061 & 0.320356215546973 & 0.320355186294337 \\
\hline 0.368077383902961 & 0.368077383877811 & 0.368076150876117 \\
\hline 0.417984229397421 & 0.417984229365926 & 0.417984229397421 \\
\hline
\end{tabular}

Example 2: [see (6)]

$\frac{\partial^{2} u}{\partial t^{2}}=\frac{\partial^{2} u}{\partial x^{2}}-u-u^{3} \quad, t>0$

With the initial conditions

$u(x, 0)=A\left[1+\cos \left[\left(\frac{2 \pi x}{L}\right)\right], u_{t}=0 \quad, 0 \leq x \leq L\right.$

The boundary conditions are given by

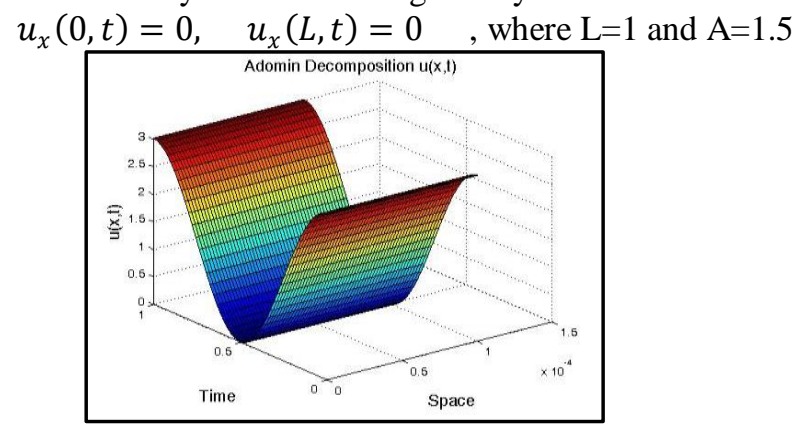

Fig. (4) ) ADM solution to $0<\mathrm{t}<0.0001$ and $0<x<1$

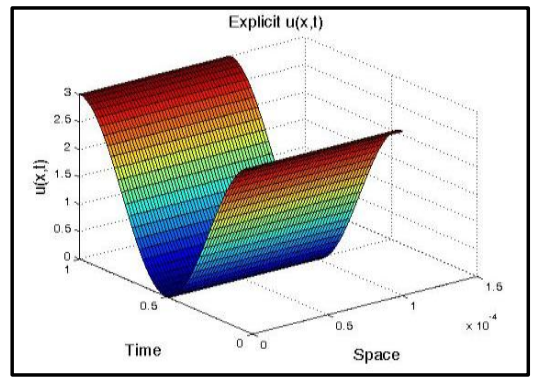

Fig. (5) EFDM solution to $0<\mathrm{t}<0.0001$ and $0<x<1$ 
Table (2) comparison between ADM, EFDM solution at $\mathrm{t}=2$ with $\mathrm{dx}=0.020408163265306$

\begin{tabular}{|l|l|}
\hline ADM & EFDM \\
\hline 2.987685020734869 & 2.987685020550971 \\
\hline 2.950942294558544 & 2.950942294379764 \\
\hline 2.806978056185084 & 2.806978056025879 \\
\hline 2.577524025146591 & 2.577524025017099 \\
\hline 2.435234702788101 & 2.435234702676172 \\
\hline 2.107175014683591 & 2.107175014609870 \\
\hline 1.739399842550069 & 1.739399842515833 \\
\hline 1.548077366357483 & 1.548077366342586 \\
\hline 1.355965461138478 & 1.355965461142287 \\
\hline 1.166218599065529 & 1.166218599087200 \\
\hline 0.981952418368039 & 0.981952418406554 \\
\hline 0.806192564638747 & 0.806192564692939 \\
\hline 0.641825009816746 & 0.641825009885315 \\
\hline 0.491548664608025 & 0.491548664689548 \\
\hline 0.357831062446298 & 0.357831062539230 \\
\hline 0.242867842662239 & 0.242867842764914 \\
\hline 0.148546698146371 & 0.148546698257009 \\
\hline 0.076416379483997 & 0.076416379600713 \\
\hline 0.027661264513402 & 0.027661264634223 \\
\hline 0.003081910874495 & 0.003081910997386 \\
\hline 0.003081910874495 & 0.003081910997386 \\
\hline 0.027661264513402 & 0.027661264634223 \\
\hline 0.076416379483997 & 0.076416379600713 \\
\hline 0.148546698146371 & 0.148546698257009 \\
\hline 0.242867842662239 & 0.242867842764914 \\
\hline 0.357831062446298 & 0.357831062539229 \\
\hline 0.491548664608024 & 0.491548664689547 \\
\hline 0.641825009816745 & 0.641825009885314 \\
\hline 0.806192564638747 & 0.806192564692939 \\
\hline 0.981952418368038 & 0.981952418406553 \\
\hline 1.166218599065527 & 1.166218599087198 \\
\hline 1.355965461138476 & 1.355965461142286 \\
\hline 1.548077366357482 & 1.548077366342585 \\
\hline 1.739399842550069 & 1.739399842515833 \\
\hline 1.926791379946547 & 1.926791379892598 \\
\hline 2.107175014683589 & 2.107175014609868 \\
\hline
\end{tabular}

Example 3: [11]

with the initial condition of

$$
u_{t}+u u_{x}+u_{x x}+u_{x x x x}=0, \quad x \in[0,32 \pi],
$$

$$
u(x, 0)=\cos \left(\frac{x}{16}\right)\left(1+\sin \frac{x}{16}\right)
$$

Exact solution of problem is given by

$$
\begin{aligned}
& u(x, t)=\cos \left(\frac{x}{16}-t\right)\left(1+\sin \left(\frac{x}{16}-t\right)\right) \\
& A_{0}=u_{0} u_{0 x}=-\cos \left(\frac{x}{16}\right)\left(1+\sin \left(\frac{x}{16}\right)\right)\left(\frac{\sin \left(\frac{x}{16}\right)\left(1+\sin \left(\frac{x}{16}\right)\right)-\cos ^{2}\left(\frac{x}{16}\right)}{16}\right) \\
& u_{1}(x, t)=t \cos \left(\frac{x}{16}\right)\left(\frac{9200 \sin \left(\frac{x}{16}\right)-8192 \cos ^{2}\left(\frac{x}{16}\right) \sin \left(\frac{x}{16}\right)-12288 \cos ^{2}\left(\frac{x}{16}\right)+8447}{65536}\right) \\
& A_{1}=u_{0} u_{1 x}+u_{1} u_{0 x}=-\left(t \cos \left(\frac{x}{16}\right)\right) \\
& \qquad\left(\frac{\left(35294 \sin \left(\frac{x}{16}\right)-118720 \cos ^{2}\left(\frac{x}{16}\right) \sin \left(\frac{x}{16}\right)+49152 \cos ^{2}\left(\frac{x}{16}\right) \sin \left(\frac{x}{16}\right)-134861 \cos ^{2}\left(\frac{x}{16}\right)+102400 \cos ^{4}\left(\frac{x}{16}\right)+35294\right)}{1048576}\right) \\
& u_{2}(x, t)=\left(t^{2} \cos \left(\frac{x}{16}\right)\right)
\end{aligned}
$$




$$
\begin{gathered}
\left(\left(\frac{165437696 \sin \left(\frac{x}{16}\right)-517734400 \cos ^{2}\left(\frac{x}{16}\right) \sin \left(\frac{x}{16}\right)+201326592 \cos ^{4}\left(\frac{x}{16}\right) \sin \left(\frac{x}{16}\right)}{8589934592}\right)\right. \\
\left.+\left(\frac{-579706880 \cos ^{2}\left(\frac{x}{16}\right)+419430400 \cos ^{4}\left(\frac{x}{16}\right)+164855297}{8589934592}\right)\right)
\end{gathered}
$$

$$
\begin{aligned}
A_{2}= & u_{0} u_{2 x}+u_{1} u_{1 x}+u_{2} u_{0 x} \\
= & -\left(t^{2} \cos \left(\frac{x}{16}\right)\right)\left(\left(\frac{972569604 \sin \left(\frac{x}{16}\right)-6823380992 \cos ^{2}\left(\frac{x}{16}\right) \sin \left(\frac{x}{16}\right)}{137438953472}\right)\right. \\
+ & \left(\frac{9043968000 \cos ^{4}\left(\frac{x}{16}\right) \sin \left(\frac{x}{16}\right)-2147483648 \cos ^{6}\left(\frac{x}{16}\right) \sin \left(\frac{x}{16}\right)}{137438953472}\right) \\
+ & \left.\left(\frac{-7304897891 \cos ^{2}\left(\frac{x}{16}\right)+12242182144 \cos ^{4}\left(\frac{x}{16}\right)-5754585088 \cos ^{6}\left(\frac{x}{16}\right)+971435586}{137438953472}\right)\right) \\
u_{3}(x, t)=\left(t^{3} \cos \left(\frac{x}{16}\right)\right) & \left(\left(\frac{4859359014912 \sin ^{2}\left(\frac{x}{16}\right)-30758081134592 \cos ^{2}\left(\frac{x}{16}\right) \sin \left(\frac{x}{16}\right)}{1688849860263936}\right)\right. \\
& +\left(\frac{\left.38638599536640 \cos ^{4}\left(\frac{x}{16}\right) \sin \left(\frac{x}{16}\right)-8796093022208 \cos ^{6}\left(\frac{x}{16}\right) \sin \left(\frac{x}{16}\right)-33071821131776 \cos ^{2}\left(\frac{x}{16}\right)\right)}{1688849860263936}\right) \\
& \left.+\left(\frac{52566188621824 \cos ^{4}\left(\frac{x}{16}\right)-23570780520448 \cos ^{6}\left(\frac{x}{16}\right)+4826353967871}{1688849860263936}\right)\right)
\end{aligned}
$$

Then approximation solution of Eq.(33) is $u(x, t)=u_{0}+u_{1}+u_{2}+u_{3}$ with third-order approximation. Now we compare exact solution with Adomain Decomposition Method (ADM) solution in Fig.6,Fig.7

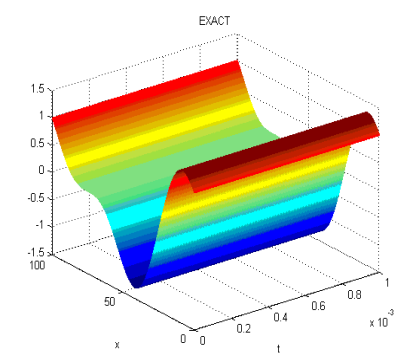

Fig.(6) Exact solution of Kuramoto-Sivashinsky equation Sivashinsky equation

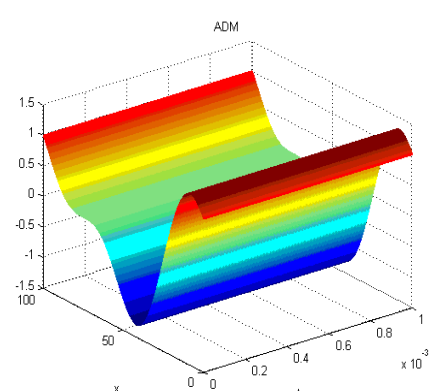

Fig.(7) ADM solution of Kuramoto- 


\begin{tabular}{|c|c|c|c|c|}
\hline$x * \pi$ & $t$ & EXACT SOLUTION & APPROXIMATION & ABSOLUTE ERROR \\
\hline \multirow{6}{*}{0} & 0 & 1.000000000000000 & 1.000000000000000 & 0 \\
\hline & 0.0002 & 0.999799980005333 & 0.999988278219568 & $1.882982142341616 \mathrm{e}-004$ \\
\hline & 0.0004 & 0.999599920042668 & 0.999976556481800 & $3.766364391323274 \mathrm{e}-004$ \\
\hline & 0.0006 & 0.999399820144005 & 0.999964834786718 & $5.650146427130798 \mathrm{e}-004$ \\
\hline & 0.0008 & 0.999199680341350 & 0.999953113134345 & $7.534327929941131 \mathrm{e}-004$ \\
\hline & 0.001 & 0.998999500666708 & 0.999941391524700 & $9.418908579912344 \mathrm{e}-004$ \\
\hline \multirow{6}{*}{6.4} & 0 & 0.602909620521184 & 0.602909620521184 & 0 \\
\hline & 0.0002 & 0.603261605525986 & 0.602924029951888 & $3.375755740976372 \mathrm{e}-004$ \\
\hline & 0.0004 & 0.603613531113795 & 0.602938440031289 & $6.750910825057410 \mathrm{e}-004$ \\
\hline & 0.0006 & 0.603965397251127 & 0.602952850759426 & $1.012546491701349 \mathrm{e}-003$ \\
\hline & 0.0008 & 0.604317203904505 & 0.602967262136334 & $1.349941768170049 \mathrm{e}-003$ \\
\hline & 0.001 & 0.604668951040459 & 0.602981674162053 & $1.687276878405974 \mathrm{e}-003$ \\
\hline \multirow{6}{*}{12.8} & 0 & (-1.284545252522524 & (-1.284545252522524 & 0 \\
\hline & 0.0002 & -1.284489444647476 & -1.284551820710811 & $6.237606333447943 e-005$ \\
\hline & 0.0004 & -1.284433528322049 & -1.284558388250076 & $1.248599280267992 \mathrm{e}-004$ \\
\hline & 0.0006 & -1.284377503541076 & -1.284564955140284 & $1.874515992073000 \mathrm{e}-004$ \\
\hline & 0.0008 & -1.284321370299405 & -1.284571521381397 & $2.501510819914454 \mathrm{e}-004$ \\
\hline & 0.001 & -1.284265128591897 & -1.284578086973377 & $3.129583814804882 \mathrm{e}-004$ \\
\hline \multirow{6}{*}{19.2} & 0 & -0.333488736227371 & -0.333488736227371 & 0 \\
\hline & 0.0002 & -0.333668118536193 & -0.333484164639820 & $1.839538963727683 \mathrm{e}-004$ \\
\hline & 0.0004 & -0.333847544554259 & -0.333479593175463 & 3.679513787964717e-004 \\
\hline & 0.0006 & -0.334027014266963 & -0.333475021834294 & $5.519924326690129 \mathrm{e}-004$ \\
\hline & 0.0008 & -0.334206527659685 & -0.333470450616308 & $7.360770433769148 \mathrm{e}-004$ \\
\hline & 0.001 & -0.334386084717798 & -0.333465879521502 & $9.202051962963198 \mathrm{e}-004$ \\
\hline \multirow{6}{*}{25.6} & 0 & 0.015124368228711 & 0.015124368228711 & 0 \\
\hline & 0.0002 & 0.015095977652350 & 0.015123677789543 & $2.770013719346869 \mathrm{e}-005$ \\
\hline & 0.0004 & 0.015067621719845 & 0.015122987353990 & $5.536563414442614 \mathrm{e}-005$ \\
\hline & 0.0006 & 0.015039300412906 & 0.015122296922050 & $8.299650914345494 \mathrm{e}-005$ \\
\hline & 0.0008 & 0.015011013713235 & 0.015121606493723 & $1.105927804877852 \mathrm{e}-004$ \\
\hline & 0.001 & 0.014982761602528 & 0.015120916069009 & $1.381544664819934 \mathrm{e}-004$ \\
\hline \multirow{6}{*}{32} & 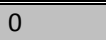 & 1.000000000000000 & 1.000000000000000 & 0 \\
\hline & 0.0002 & 0.999799980005333 & 0.999988278219567 & $1.882982142346057 \mathrm{e}-004$ \\
\hline & 0.0004 & 0.999599920042668 & 0.999976556481800 & $3.766364391322163 e-004$ \\
\hline & 0.0006 & 0.999399820144005 & 0.999964834786718 & $5.650146427135239 \mathrm{e}-004$ \\
\hline & 0.0008 & 0.999199680341350 & 0.999953113134344 & 7.534327929941131e-004 \\
\hline & 0.001 & 0.998999500666708 & 0.999941391524699 & $9.418908579915675 \mathrm{e}-004$ \\
\hline
\end{tabular}

\section{Conclusion}

Adomain Decomposition Method used to solve nonlinear partial differential equation. Figures and tables shows that the comparison between the exact solution and the numerical solution obtained by Adomain Decomposition Method (ADM). It can be seen that the solution obtained by the present method is nearly identical with that given by exact solution. The absolute error of example be observed and showed that the ADM is closed to the exact solution; also this method is suitable for this kind of problem, And powerful mathematical tool for solving nonlinear problems in science and engineering.

\section{References}

[1] Jerri, Introduction To Integral Equations With Applications, (Marcel Dekker, Inc, New York and Basel,1985)

[2] Raftari," Application of He's homotopy perturbation method and variational iteration method for nonlinear partial integrodifferential equations", World Applied Sciences Journal, 7, 4, (2009), 399-404.

[3] Raftari," Numerical solutions of the linear volterra integro-differential equations: homotopy perturbation method and finite Difference method", World Applied Sciences Journal, 9, (2010), 7-12.

[4] A., Coddinggton, An introduction To Ordinary Differential Equations With Applications, (Prentics-Hall,INC., 1961) .

[5] Brauer and J. A Nohel, Ordinary Differential Equations, ( a First Course, $2^{\text {nd }}$ edition, W. A. Benjamin,

[6] Nicolis. and Prigogine I.,(1977), Self-Organization in Nonequilibrium Systems, Wiley, New York. INC. London, 1973).

[7] J. H Mathews. and K. D.Fink, (1999), Numerical Methods Using Matlab, Prentice- Hall, Inc.

[8] J.Crank," The Mathematics of Diffusion “, Oxford University Press, Oxford, (1975).

[9] J.D Murray.,(1993), Mathematical Biology,2nd ed. Springer-Verlag Berlin.

[10] J.D. Cole," Perturbation Method in Applied Mathematics", Blaisdell, Waltham, MA, (1968).

[11] J.D. Murray,” Mathematical Biology “, Springer, Berlin, (1989).

[12] J.H. He," Homotopy perturbation technique", Comput. Methods Appl, Mech, Engrg, 178, (1999), $257-262$.

[13] L.Debnath, Nonlinear Partial Differential Equations for Scientists and Engineers, (Birkhauser, Boston, ,1997).

[14] M. Ayub,A. Rasheed,T. Hayat," Exact flow of a third grade fluid past a porous plate using homotopy analysis”, Int. J. Eng. Sci, 41, (2003), 2091-2103.

[15] M. Hayat,M. Khan," Homotopy solutions for a generalized second grade fluid past a porous plate", Nonlinear Dynam, 42, (2005), 395-405.

[16] M. Matinfar,M. Saeidy," The homotopy perturbation method for solving higher dimensional initial boundary value problems of variable coefficients", World Applied Sciences Journal, 5, 1, (2009),72-80. 
[17] M. Sajad,T. Hayat,S. Asghar," On the analytic solution of the steady flow of a fourth grade fluid. Phys.”, Lett. A, 355, (2006), 1826.

[18] M. Sajid,T. Hayat,S. Asghar," Non-similar solution for the ax symmetric flow of a third-grade fluid over radially stretching sheet ", Acta Mechanica,189, (2007), 193-205.

[19] M. Shanthakumar, (1989), Computer Based Numerical Analysis, Khanna publisher, Neisaraic Delhi-110006 India.

[20] M.A. El-Tawi,H.N.Hassan," A new application of using homotopy analysis method for solving stochastic quadratic nonlinear diffusion equation "Int. J. of Appl Math and Mech, 9, 16, (2013), 35-55.

[21] R. Aris," The Mathematical Theory of Diffusion and Reaction in Permeable Catalysts “, Oxford University Press, Oxford, (1975).

[1] R. Cherniha,M. Servo," Lie and non-lie symmetries of nonlinear di_usion equations with convection term ", Symmetry in Nonlinear Mathematical Physics, 2, (1997), 444-449.

[22] R. K., Saeed, Computational methods for solving system of linear Volterra and integro-differential equations, Ph.D. Thesis, Salahaddin University/Erbil, Iraq, 2006.

[23] R. Temam, (1993), Infinite dimensional Dynamical systems in chanics and physics, Springer-Verlag,berlin.

[24] S.J. Liao," Beyond Perturbation: Introduction to the Homotopy Analysis Method", Chapman and Hall/CRC Press, Boca Raton, (2003).

[25] S.J. Liao," Notes on the homotopy analysis method: some definitions and theories ", Communications in Nonlinear Science Numerical Simulation, 14, (2009), 983-997.

[26] S.J. Liao," On the homotopy analysis method for nonlinear problems", App. Math. Comput. 147, (2004), $499-513$.

[27] S.J. Liao," The proposed homotopy analysis method techniques for the solution of nonlinear problems", Thesis Shanghai, Jiao Tong University Shanghai, (1992).

[28] T. Hayat,M. Khan,” Exact flow of a third grade fluid past a porous plate using homotopy analysis”, Int. J. Eng. Sci, 56, (2005), 1012-1029.

[29] V.F Zaitsev and A.D. Polyanim, Handbook of Nonlinear Partial Differential Equations,(Chapman and Hall/CRC Press, Boca Raton, 2004).

[30] W.F. Ames,” Nonlinear Partial Differential Equations in Engineering “, Academic Press, New York, (1972).

[31] Y. Mahmoudi,E.M. Kazemian," The Homotopy Analysis Method for Solving the Kuramoto-Tsuzuki Equation ",World Applied Sciences Journal, 21, 12, (2013), 1776-1781.

[32] Y. Yuncheng, ( 2007), Global Dynamics of the Brusselator Equations, Dynamics of PDE, Vol.4, No.2, 167-1 